\title{
Cerebral Tissue Oxygenation and Regional Oxygen Saturation Can Be Used to Study Cerebral Autoregulation in Prematurely Born Infants
}

ALEXANDER CAICEDO, DOMINIQUE DE SMET, GUNNAR NAULAERS, LIEVEKE AMEYE, JOKE VANDERHAEGEN, PETRA LEMMERS, FRANK VAN BEL, AND SABINE VAN HUFFEL

\begin{abstract}
Department of Electrical Engineering (ESAT) [A.C., D.S., L.A., S.H.], Katholieke Universiteit Leuven, and IBBT-K.U.Leuven Future
Health Department, Leuven 3001, Belgium; Neonatal Intensive Care Unit [G.N., J.V.], University Hospital Gasthuisberg, Katholieke

Universiteit Leuven, Leuven 3000, Belgium; Department of Neonatology [P.L., F.B.], Wilhelmina Children's Hospital, University Medical
\end{abstract}

Centre, Utrecht 3584 CX, The Netherlands

\begin{abstract}
The coupling of cerebral intravascular oxygenation $(\mathrm{dHbD})$ with mean arterial blood pressure (MABP) was taken as a reflection of autoregulation assuming constant arterial oxygen content. However, this method is sensitive to movement artifacts. We examined whether the cerebral tissue oxygenation index (cTOI) and regional oxygen saturation $\left(\mathrm{rScO}_{2}\right)$ may replace $\mathrm{dHbD}$ and changes in total $\mathrm{Hb}(\mathrm{dHbT})$, respectively. Correlation $(\mathrm{COR})$ and coherence $(\mathrm{COH})$ were used to measure the agreement of MABP with $\mathrm{rScO}_{2} / \mathrm{dHbT}$ and $\mathrm{cTOI} / \mathrm{dHbD}$. $\mathrm{dHbD} / \mathrm{cTOI}$ and $\mathrm{dHbT} / \mathrm{rScO}_{2}$ recordings of, respectively, 34 and 20 preterm infants in need for intensive care were studied during the first days of life. $\mathrm{dHbD}$ and cTOI were obtained with the NIRO300 and $\mathrm{rScO}_{2}$ and $\mathrm{dHbT}$ with the INVOS4100. Invasive MABP was measured continuously. $\mathrm{COR}$ and $\mathrm{COH}$ scores of MABP versus $\mathrm{dHbD} /$ $\mathrm{dHbT}$ were compared with the corresponding ones by replacing $\mathrm{dHbD} /$ $\mathrm{dHbT}$ by $\mathrm{cTOI} / \mathrm{rScO}_{2}$, respectively. Generally, no significant score differences were found for $\mathrm{dHbD} / \mathrm{cTOI}$. Differences for $\mathrm{dHbT} / \mathrm{rScO}_{2}$ were slightly larger but still within the normal variation of the parameters. Differences become insignificant when restricting calculations to epochs of larger variation in MABP $(>10 \mathrm{~mm} \mathrm{Hg})$. Hence, we suggest that cTOI and $\mathrm{rScO}_{2}$ can be used to study cerebral autoregulation in newborns. (Pediatr Res 69: 548-553, 2011)
\end{abstract}

$\mathrm{L}$ assen (1) was the first to describe cerebral autoregulation in man. Cerebral autoregulation is a property of arteries in the brain to constrict in response to an increase in transmural blood pressure and to dilate in response to a decrease in blood pressure, with the effect of keeping blood flow more or less constant within a range of arterial blood pressures. This response has a limited capacity and as a result blood flow will decrease or increase when blood pressure decreases below a lower threshold or increases above an upper threshold, respectively (2). Pryds et al. (3) described a loss of autoregulation in very sick preterm infants, which resulted in the hypothesis that there was a loss of autoregulation in preterm infants and that controlling blood pressure would prevent cerebral damage. However, there is no good evidence that a correlation (COR) between blood pressure and

Received July 22, 2010; accepted December 14, 2010.

Correspondence: Sabine Van Huffel, Ph.D., Division SCD, Department of Electrical Engineering (ESAT), Katholieke Universiteit Leuven, Kasteelpark Arenberg 10, bus 2446, Leuven 3001; e-mail: sabine.vanhuffel@esat.kuleuven.be

Supported by Research Council KUL: GOA AMBioRICS, GOA MANET, CoE EF/05/006, by FWO projects G.0519.06 (noninvasive brain oxygenation) and G.0341.07 (Data fusion), and by Belgian Federal Science Policy Office IUAP P6/04 (DYSCO). cerebral damage exists, indicating that autoregulation is more complex in premature infants than originally thought (4). Even with very low blood pressures, normal cerebral blood flow (CBF) was described in very LBW infants (5). Both dynamic and static autoregulation are described. Dynamic autoregulation describes the response of autoregulation within 5 to $10 \mathrm{~s}$ after a change in blood pressure and can be assessed noninvasively with Doppler ultrasound (6-8) and near-infrared spectroscopy (NIRS) (9). Tsuji et al. (10) were the first to report on the use of NIRS to assess autoregulation. In a previous study (11), the authors validated changes in the cerebral intravascular oxygenation $(\mathrm{dHbD})$ as a measure of $\mathrm{CBF}$ in an experimental model. $\mathrm{dHbD}$, representing the difference between changes in oxygenated $\left(\mathrm{dHbO}_{2}\right)$ and reduced $(\mathrm{dHbR}) \mathrm{Hb}$, can be continuously measured by means of any NIRS device providing the raw optical densities and slope data. Tsuji et al. (10) found a high COR between $\mathrm{dHbD}$ and mean arterial blood pressure (MABP) changes, indicating loss in autoregulation. A high COR was found between intact autoregulation and outcome, i.e. the frequency of severe intraventricular bleedings. Moreover, Wong et al. (12) found that high coherence $(\mathrm{COH})$ between MAP and TOI indicates impaired cerebral autoregulation in clinically sick preterm infants and is strongly associated with subsequent mortality. However, $\mathrm{dHbD}$ is a relative parameter and difficult to measure because of movement artifacts. If we want to have a continuous robust measurement, other parameters of oxygenation should be used. The cerebral tissue oxygenation index (cTOI) and the cerebral regional oxygen saturation $\left(\mathrm{rScO}_{2}\right)$ are two promising NIRS parameters that provide absolute values of regional $\mathrm{Hb}$ oxygen saturation in absolute terms. In addition, they are less prone to movement artifacts (13). cTOI is based on spatially resolved spectroscopy and calculated by $\left(\mathrm{kHbO}_{2} / \mathrm{kHbT}\right) \times 100(\%)$, where $\mathrm{HbO}_{2}, \mathrm{HbR}$, and $\mathrm{HbT}$ denote, up to an unknown scaling factor $\mathrm{k}$, the absolute

\footnotetext{
Abbreviations: CBF, cerebral blood flow; COH, coherence; COR, correlation; cTOI, cerebral tissue oxygenation index; $\mathbf{d H b D}$, cerebral intravascular oxygenation; $\mathbf{d H b O}_{2}$, changes in oxygenated $\mathrm{Hb}$; $\mathbf{d H b R}$, changes in reduced $\mathrm{Hb}$; dHbT, changes in total $\mathrm{Hb}$; $\mathbf{H b O}_{2}$, oxygenated $\mathrm{Hb}$; $\mathbf{H b T}$, total $\mathrm{Hb}$; MABP, mean arterial blood pressure; NIRS, near-infrared spectroscopy; PMA, postmenstrual age; $\mathbf{r S c O}_{2}$, cerebral regional oxygen saturation; $\mathbf{S a O}_{2}$, arterial oxygen saturation
} 
concentration of, respectively, oxygenated $\mathrm{Hb}$, reduced $\mathrm{Hb}$, and total $\mathrm{Hb}\left(\mathrm{HbT}=\mathrm{HbO}_{2}+\mathrm{HbR}\right)(14) \cdot \mathrm{rScO}_{2}$ is calculated by a proprietary algorithm, which is also based on spatially resolved spectroscopy (15). Both cTOI and $\mathrm{rScO}_{2}$ are absolute values and have been validated against the jugular venous saturation and each other $(16,17)$. In this study, we examined whether cTOI and $\mathrm{rScO}_{2}$ can replace $\mathrm{dHbD}$ and $\mathrm{dHbT}$, respectively, in the measurement of autoregulation.

\section{METHODS}

A total of 54 infants with need for intensive care were monitored during the first days of life at the NICU of two different hospitals. Their recordings were collected in three different datasets. In the first dataset, a total of 14 infants from the NICU of the University Hospital Leuven, Belgium, were included. These infants with a mean postmenstrual age (PMA) of 37 6/7 wk (27-47) and a mean body weight of $2931 \mathrm{~g}$ (855-4380) were treated with propofol to attain a short-lasting sedation during elective chest tube removal to facilitate chest tube removal and avoid external aspiration of air. This dataset, called the propofol dataset in this article, was also used in a separate study presented in Ref. 18. The second dataset contains the recordings of 20 prematurely born infants with need for intensive care that were monitored during the first $3 \mathrm{~d}$ of life at the same NICU of the University Hospital Leuven, and it will be referred to as the Leuven dataset. Mean PMA of the Leuven infants was $285 / 7$ wk (SD $\pm 32 / 7$ ), mean body weight was $1125 \mathrm{~g}(\mathrm{SD} \pm 503.76)$, and mean postnatal age (PNA) at the time of measurements was $1.4 \mathrm{~d}(\mathrm{SD} \pm 1.06)$. Cranial ultrasounds are performed at $\mathrm{d} 1$, 3 , and 7 or more frequently if needed.

The third dataset includes the recordings of 20 prematurely born infants monitored at the NICU of the University Medical Centre Utrecht, The Netherlands. These infants had a mean PMA of 29 2/7 wk (SD $\pm 12 / 7)$, mean PNA at the time of measurements $2 \mathrm{~d}(\mathrm{SD} \pm 1)$, and a mean body weight of $1114 \mathrm{~g}$ (SD \pm 316.94). This dataset will be referred to as the Utrecht dataset. Demographic and clinical data for all infants are summarized in Table 1. Cranial ultrasounds are performed at d 1,2,3, and then weekly.

The medical ethical committee of the University Hospital Gasthuisberg in Leuven and the Wilhelmina Children's Hospital in Utrecht approved this study. Informed parental consent was obtained in all cases.

Vital signs. Arterial oxygen saturation $\left(\mathrm{SaO}_{2}\right)$ was continuously recorded by pulse oxymetry on a limb and MABP by an indwelling arterial catheter (umbilical, tibial, or radial artery).

Cerebral hemodynamics. In Leuven, the NIRO300 device (Hamamatsu, Japan) was used for the noninvasive monitoring of cerebral hemodynamic and oxygenation parameters cTOI, $\mathrm{dHbO}_{2}$, and $\mathrm{dHbR}$. The differential path length factor (taking into account the scattering of the near-infrared light into the brain) was set at $4.39(19,20)$ and encoded into the computer as a constant value. For calculation of cTOI, the absorption of near-infrared light is measured at three points, and the diffusion equation is used. $\mathrm{dHbD}$ was calculated afterward as the difference between $\mathrm{dHbO}_{2}$ and $\mathrm{dHbR}$.

In Utrecht, with the INVOS4100 instrument (Somanetics Corp, Troy, MI), $\mathrm{rScO}_{2}$ and the optical densities at a distance $4 \mathrm{~cm}$ (deep signal) and $3 \mathrm{~cm}$ (shallow signal) from the detector were recorded simultaneously. For the calculation of $\mathrm{rScO}_{2}$, the scattering of near-infrared light at two wavelengths, namely 730 and $810 \mathrm{~nm}$, is measured at $3 \mathrm{~cm}$ and deducted from the measurement at the second optode at $4 \mathrm{~cm}$. dHbT was computed afterward as the inverse of the difference between deep and shallow OD signals at $810 \mathrm{~nm}(21)$.
Signal processing. $\mathrm{MABP}$ and $\mathrm{SaO}_{2}$ data in Leuven were generated at $2 \mathrm{~Hz}$ and recorded at a sampling frequency of $100 \mathrm{~Hz}$ by a data acquisition system CODAS (Dataq Instruments) and stored on a computer. The NIRO 300 signals are digital and recorded with a sampling frequency of $6 \mathrm{~Hz}$. They were converted to analog signals with a sample-and-hold function before being introduced in the CODAS system (Dataq Instruments). To ensure the best comparability between both medical centers, we downsampled these signals to the same frequency of $0.0167 \mathrm{~Hz}$ (periodicity $60 \mathrm{~s}$ ). This low frequency was dictated by the sampling frequency of the $\mathrm{dHbT}$ signal (one sample per minute). Moreover, to reduce the loss of information in the new downsampled signal, the signals were first filtered with a mean average filter. In addition, to study the influence of the sampling frequency on the scores, we also analyzed the NIRO data after filtering and downsampling to $0.333 \mathrm{~Hz}$ (periodicity $3 \mathrm{~s}$ ).

In Utrecht, $\mathrm{MABP}, \mathrm{SaO}_{2}$, and $\mathrm{rScO}_{2}$ were collected simultaneously by the Poly5 system (Inspektor Research Systems, The Netherlands) with a sampling frequency of $10 \mathrm{~Hz}$ and stored on a personal computer for offline analysis. The $\mathrm{rScO}_{2}$ was recorded using the INVOS4100 with a sampling frequency of 0.25 $\mathrm{Hz}$. The signal was then converted to an analog signal with a sample-and-hold function before being introduced in the Poly5 system. Because the optical densities were sampled at $0.0167 \mathrm{~Hz}$ (i.e. one value per minute) on a separate disk drive, all signals were filtered with a mean average filter and then downsampled to this lower frequency to avoid loss of information in the new downsampled signal. By adopting this new sample frequency, we stay in accordance with the findings of von Siebenthal et al. (22) concerning the periodicity of the studied signals. A similar study of the influence of the sampling frequency on the scores, as with the NIRO300 data, was not possible with the INVOS4100 data because of the low sample frequency of dHbT.

To all data from both centers, a preprocessing algorithm was applied to remove measurement artifacts induced by medical interferences as follows. First, as described by Soul et al. (23), each artifact point from the hemodynamic data was removed. To remove occasional artifacts such as movements and displacements in the baseline, we developed a novel robust function estimator described in Ref. 24. This algorithm, programmed in Matlab (Mathworks, Natick, MA), trained a Least Squares Support Vector Machine (LS-SVM) to interpolated data as long as the duration of the artifact was shorter than $30 \mathrm{~s}$ (25), otherwise the signal was truncated. Hence, a continuous recording was divided in smaller segments free of artifacts. Only segments with length longer than $40 \mathrm{~min}$ were kept for further analysis. In addition, we divided the signal in nonoverlapping 20-min epochs. Next, we kept the signals in normal physiological ranges, particularly $\mathrm{SaO}_{2}$ in the range $87-95 \%$ (in this way the condition of constant $\mathrm{SaO}_{2}$ is nearly satisfied). Finally, we deleted remaining artifact spikes, which could not be detected in the previous step. Figure 1 displays typical recordings of $\mathrm{SaO}_{2}, \mathrm{MABP}, \mathrm{dHbD}$, and cTOI as measured on a preterm infant in Leuven.

$\mathbf{C O H}$ and $\mathbf{C O R}$. COR has been absolute valued such that its value matches the interval $[0,1](0-100 \%)$ instead of $[-1,1]$ for comparability with the $\mathrm{COH}$ method. We computed $\mathrm{COH}$ using the Welch's averaged periodogram method (26). The COR and the average of $\mathrm{COH}$ over the frequency band 0.0042 $0.00837 \mathrm{~Hz}$ (periodicity in the range 240-120 s) for the $60 \mathrm{~s}$ data, and over the frequency band $0.0033-0.04 \mathrm{~Hz}$ for the $3 \mathrm{~s}$ data (range $300-25 \mathrm{~s}$, because of the use of an antialiasing low-pass filter with cutoff frequency of $0.04 \mathrm{~Hz}$ ) (23), were used as scores for the considered signal epoch (22,27-29). Because the $\mathrm{COR}$ and $\mathrm{COH}$ values between the signals might vary as a function of time, a sliding window approach was used. Both scores were calculated over 20-min epochs. To calculate the amplitude of the $\mathrm{COH}$, the autopower and crosspower spectra densities were estimated using the Welch's averaged periodogram method. In this method, each 20 -min epoch was subdivided into five segments of duration 10 min with an overlap of $7.5 \mathrm{~min}$.

Table 1. Characteristics for the Leuven, Utrecht, and Propofol dataset

\begin{tabular}{lccc}
\hline \multicolumn{1}{c}{ Clinical characteristic } & Leuven dataset & Utrecht dataset & Propofol dataset \\
\hline Body weight $(\mathrm{g})$ & $1007(570-2935)$ & $1025(640-1690)$ & $2931(855-4380)$ \\
GA (wk) & $292 / 7(24-39)$ & $293 / 7(26-31)$ & $353 / 7(26-40)$ \\
Male/female & $10 / 10$ & $10 / 10$ & $16 / 4$ \\
Apgar 1 & $7(0-9)$ & $6(1-9)$ & $7(4-9)$ \\
Apgar 5 & $9(1-10)$ & $8.5(4-10)$ & $8(4-10)$ \\
IVH, $n(\%)$ & $7(35)$ & $4(20)$ & $0(0)$ \\
PVL, $n$ (\%) & $7(35)$ & $1(5)$ & $0(0)$ \\
Neonatal mortality, $n(\%)$ & $1(5)$ & $1(5)$ & $0(0)$ \\
Number of 20-min epochs & $4(1-15)$ & $12(4-36)$ & $1(1-2)$ \\
\hline
\end{tabular}

Median (range) unless otherwise stated.

$\mathrm{IVH}$, intraventricular hemorrhage; PVL, periventricular leukomalacia. 

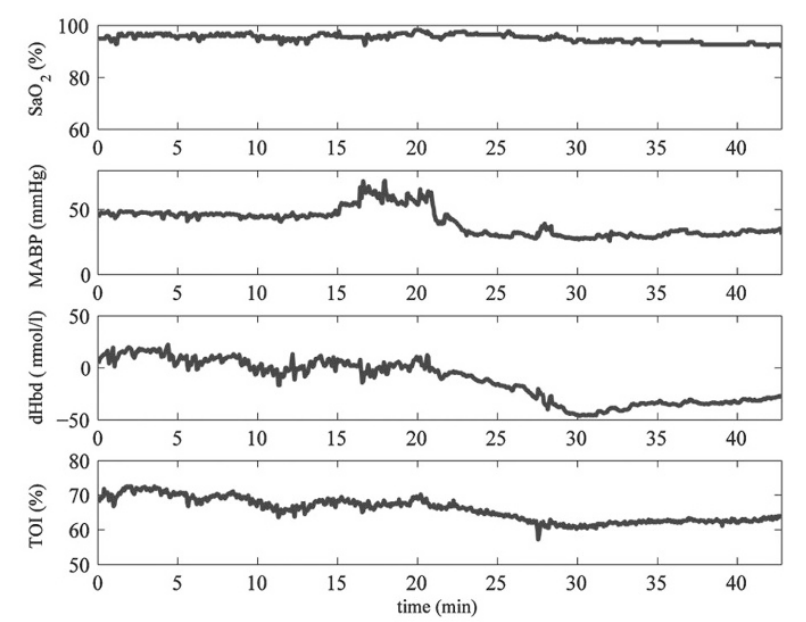

Figure 1. Typical recordings of $\mathrm{SaO}_{2}, \mathrm{MABP}, \mathrm{dHbD}$, and cTOI as measured on a preterm infant in Leuven.

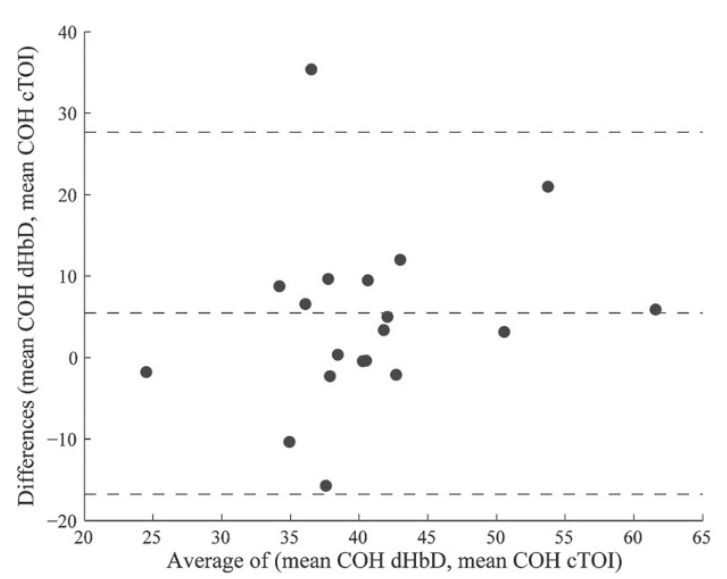

Figure 2. Bland-Altman plot for $\mathrm{COH}$ scores (dHbD/MABP and cTOI/MABP), averaged per child, in the Leuven dataset sampled at $60 \mathrm{~s}$. The central line corresponds to the mean difference and the lower and upper line to \pm 1.96 the SD.

Statistical analyses. The scores computed from cTOI versus MABP (method 1) and $\mathrm{dHbD}$ versus MABP (method 2) can be considered as two measurements from the same underlying process. Similarly, the scores computed from $\mathrm{rScO}_{2}$ versus MABP (method 1) and $\mathrm{dHbT}$ versus MABP (method 2), using the recordings from Utrecht, can be considered as two measurements of the same process. Two different analyses were performed on patient level (with one mean value per patient) and epoch level (with one score value for each 20-min epoch). On the patient level, the scores were averaged for babies of whom multiple 20-min epochs were available, to obtain one mean score value per baby. The paired $t$ test and Wilcoxon signed-rank test were applied to investigate the difference in the mean COR and mean $\mathrm{COH}$ score between 1) cTOI/MABP and $\mathrm{HbD} / \mathrm{MABP}$ and 2) $\mathrm{rScO}_{2} / \mathrm{MABP}$ and $\mathrm{dHbT} / \mathrm{MABP}$. On the epoch level, generalized linear mixed models were used to take into account the scores over all 20-min epochs (multiple measurements) per baby. In addition, to study the influence of the variations in MABP in cerebral autoregulation assessment, the scores corresponding to epochs with high variations in MABP were analyzed separately (MABP $>10 \mathrm{~mm} \mathrm{Hg}$ ). Bland-Altman plots were constructed to visualize the agreement between the two methods, as shown in Figs. 2-4. All reported $p$-values were two-tailed and we considered as statistically significant a nominal $p$-value $<0.05$. The statistical analyses were performed using the SAS system (version 9.1; SAS Institute Inc., Cary, NC).

\section{RESULTS}

Mean recording time for the propofol, Leuven, and Utrecht dataset was, respectively, $1 \mathrm{~h}$ and $32 \mathrm{~min}(\mathrm{SD} \pm 42 \mathrm{~min}$ ), $1 \mathrm{~h}$

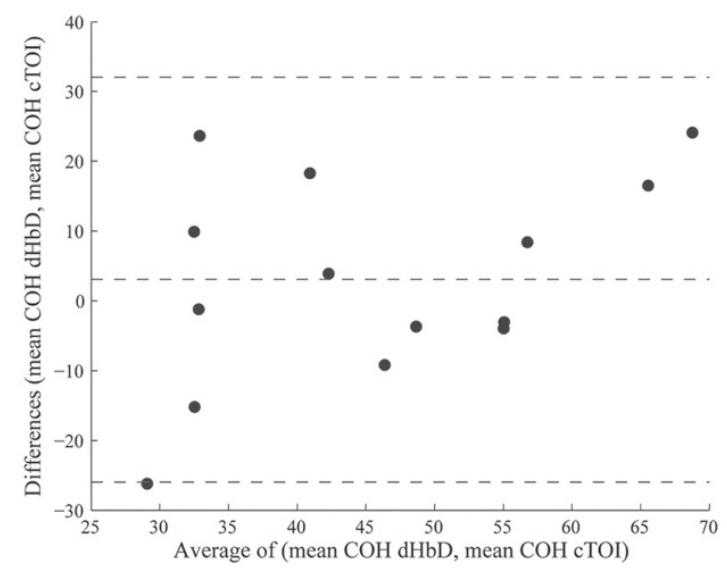

Figure 3. Bland-Altman plot for $\mathrm{COH}$ scores (dHbD/MABP and cTOI/ MABP), averaged per child, in the propofol dataset sampled at $60 \mathrm{~s}$. The central line corresponds to the mean difference and the lower and upper line to \pm 1.96 the SD.

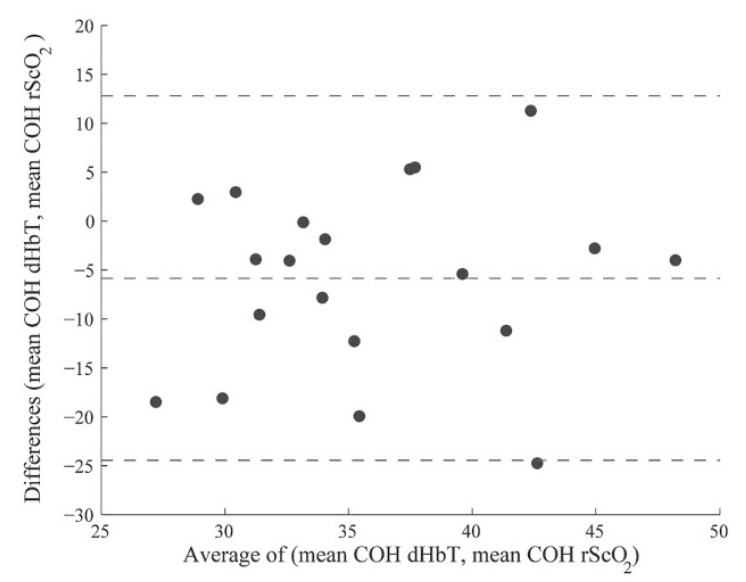

Figure 4. Bland-Altman plot for $\mathrm{COH}$ scores (dHbT/MABP and $\mathrm{rScO}_{2} /$ MABP), averaged per child, in the Utrecht dataset sampled at $60 \mathrm{~s}$. The central line corresponds to the mean difference and the lower and upper line to \pm 1.96 the SD.

and $48 \mathrm{~min}(\mathrm{SD} \pm 70 \mathrm{~min})$, and $05 \mathrm{~h}$ and $25 \mathrm{~min}(\mathrm{SD} \pm 3 \mathrm{~h}$ and $13 \mathrm{~min}$ ).

Analysis including mean COR and mean $\mathrm{COH}$ scores per patient. In Table 2, the differences in mean scores computed from $\mathrm{dHbD}$ (respectively, $\mathrm{dHbT}$ ) versus MABP (method 1) compared with cTOI (respectively, $\mathrm{rScO}_{2}$ ) versus MABP (method 2) were assessed. All data were sampled at $60 \mathrm{~s}$. For the propofol dataset, mean $\mathrm{COR}$ and mean $\mathrm{COH}$, computed from $\mathrm{dHbD}$ versus MABP compared with cTOI versus MABP, were not statistically different $(p=0.22$ and $p=0.45$, respectively). For the Leuven dataset, the corresponding mean COR scores were not statistically different $(p=0.40)$, whereas the corresponding mean $\mathrm{COH}$ scores were statistically different $(p=0.04)$ with a difference of $5.5 \%$. However, these differences remain clinically unimportant and fall within the normal variation of the parameters. This implies that both methods can be used interchangeably.

For the Utrecht dataset, mean $\mathrm{COR}$ and mean $\mathrm{COH}$ scores, computed from dHbT versus MABP compared with $\mathrm{rScO}_{2}$ 
Table 2. COR and COH scores computed from $\mathrm{dHbD}$ (respectively, dHbT) vs MABP (method 1) compared with cTOI (respectively, $r \mathrm{ScO}_{2}$ ) vs $\mathrm{MABP}($ method 2)

\begin{tabular}{|c|c|c|c|c|c|}
\hline & \multirow[b]{2}{*}{$p$} & \multicolumn{2}{|c|}{ Method 1} & \multicolumn{2}{|c|}{ Method 2} \\
\hline & & Mean $\pm \mathrm{SD}$ & $\begin{array}{c}\text { Median } \\
\text { (minimum-maximum) }\end{array}$ & Mean $\pm \mathrm{SD}$ & $\begin{array}{c}\text { Median } \\
\text { (minimum-maximum) }\end{array}$ \\
\hline \multicolumn{6}{|l|}{ Propofol } \\
\hline COR score & 0.22 & $45.6 \pm 21.0$ & $43.1(14.9-82.2)$ & $39.1 \pm 17.2$ & $35.7(12.6-69.4)$ \\
\hline $\mathrm{COH}$ score & 0.45 & $47.2 \pm 17.4$ & $45.8(16.0-80.9)$ & $44.2 \pm 12.1$ & $46.3(21.1-57.3)$ \\
\hline \multicolumn{6}{|l|}{ Leuven } \\
\hline COR score & 0.40 & $42.2 \pm 10.2$ & $40.1(29.1-67.1)$ & $39.7 \pm 10.4$ & $38.2(19.3-59.9)$ \\
\hline $\mathrm{COH}$ score & 0.04 & $44.2 \pm 11.3$ & $42.1(23.7-64.5)$ & $38.7 \pm 8.5$ & $39.8(18.9-58.6)$ \\
\hline \multicolumn{6}{|l|}{ Utrecht } \\
\hline COR score & $<0.01$ & $32.4 \pm 5.0$ & $33.8(22.6-42.4)$ & $41.7 \pm 7.6$ & $40.0(31.5-65.6)$ \\
\hline $\mathrm{COH}$ score & 0.01 & $33.0 \pm 7.9$ & $31.3(18.0-48.0)$ & $38.8 \pm 7.0$ & $36.6(27.8-55.0)$ \\
\hline
\end{tabular}

All data were sampled at $60 \mathrm{sec}$ and scores given in percentage.

Table 3. $C O R$ and $C O H$ scores computed from $d H b D$ (respectively, dHbT) vs MABP (method 1) compared with cTOI (respectively, $r \mathrm{ScO}_{2}$ ) vs $\mathrm{MABP}$ (method 2) for all epochs per patient

\begin{tabular}{|c|c|c|c|c|c|}
\hline & \multirow[b]{2}{*}{$p$} & \multicolumn{2}{|c|}{ Method 1} & \multicolumn{2}{|c|}{ Method 2} \\
\hline & & Mean $\pm \mathrm{SD}$ & $\begin{array}{c}\text { Median } \\
\text { (minimum-maximum) }\end{array}$ & Mean $\pm \mathrm{SD}$ & $\begin{array}{c}\text { Median } \\
\text { (minimum-maximum) }\end{array}$ \\
\hline \multicolumn{6}{|c|}{ Propofol $60 \mathrm{~s}$ data $(n=53,14$ babies $)$} \\
\hline COR score & 0.21 & $46.5 \pm 29.8$ & $44.6(0.1-99.8)$ & $39.8 \pm 27.6$ & $34.2(0.8-88.3)$ \\
\hline $\mathrm{COH}$ score & 0.38 & $48.0 \pm 22.4$ & $47.9(8.3-95.4)$ & $44.7 \pm 20.2$ & $43.5(4.1-91.0)$ \\
\hline \multicolumn{6}{|c|}{ Leuven $60 \mathrm{~s}$ data $(n=284,20$ babies $)$} \\
\hline COR score & 0.95 & $43.1 \pm 24.1$ & $40.5(0.8-94.7)$ & $43.2 \pm 23.2)$ & $43.3(0.2-94.7)$ \\
\hline $\mathrm{COH}$ score & 0.08 & $42.8 \pm 24.5$ & $39.9(2.2-96.8)$ & $39.3 \pm 22.1$ & $38.2(0.9-93.9)$ \\
\hline \multicolumn{6}{|c|}{ Utrecht 60 seconds data ( $n=342,20$ babies) } \\
\hline COR score & $<0.01$ & $31.9 \pm 21.0$ & $28.6(0.1-82.3)$ & $41.6 \pm 25.0$ & $41.9(0.04-95.8)$ \\
\hline $\mathrm{COH}$ score & $<0.01$ & $31.3 \pm 19.3$ & $29.8(0.5-87.1)$ & $38.8 \pm 23.3$ & $35.9(0.6-97.8)$ \\
\hline
\end{tabular}

The scores are given in percentage. The number of considered 20-min epochs is denoted by $n$.

Table 4. $C O R$ and $C O H$ scores computed from $d H b D$ (respectively, dHbT) vs MABP (method 1) compared with cTOI (respectively, $\mathrm{rScO}_{2}$ ) vs $\mathrm{MABP}$ (method 2) for 20-min epochs with variations in $\mathrm{MABP}>10 \mathrm{~mm} \mathrm{Hg}$

\begin{tabular}{|c|c|c|c|c|c|}
\hline & \multirow[b]{2}{*}{$p$} & \multicolumn{2}{|c|}{ Method 1} & \multicolumn{2}{|c|}{ Method 2} \\
\hline & & Mean $\pm \mathrm{SD}$ & $\begin{array}{c}\text { Median } \\
\text { (minimum-maximum) }\end{array}$ & Mean $\pm \mathrm{SD}$ & $\begin{array}{c}\text { Median } \\
\text { (minimum-maximum) }\end{array}$ \\
\hline \multicolumn{6}{|c|}{ Propofol $60 \mathrm{~s}$ data $(n=14,8$ babies $)$} \\
\hline COR score & 0.96 & $55.2 \pm 28.4$ & $55.0(12.7-99.8)$ & $54.7 \pm 26.3$ & $69.5(9.3-83.4)$ \\
\hline $\mathrm{COH}$ score & 0.76 & $44.0 \pm 23.9$ & $42.3(8.3-95.4)$ & $41.7 \pm 23.5$ & $39.4(13.0-91.0)$ \\
\hline \multicolumn{6}{|c|}{ Leuven 60 seconds data ( $n=46,15$ babies) } \\
\hline COR score & 0.57 & $47.1 \pm 26.7$ & $50.2(1.3-92.7)$ & $44.2 \pm 25.5$ & $46.8(3.7-93.1)$ \\
\hline $\mathrm{COH}$ score & 0.68 & $46.1 \pm 27.8$ & $45.0(4.0-96.8)$ & $43.8 \pm 25.3$ & $44.0(3.3-91.3)$ \\
\hline \multicolumn{6}{|c|}{ Utrecht $60 \mathrm{~s}$ data ( $n=62,15$ babies) } \\
\hline COR score & 0.09 & $35.4 \pm 22.9$ & $35.2(0.1-74.7)$ & $43.1 \pm 27.0$ & $42.0(0.04-92.5)$ \\
\hline $\mathrm{COH}$ score & 0.21 & $35.3 \pm 20.7$ & $33.8(0.8-82.6)$ & $40.3 \pm 25.9$ & $37.7(1.1-97.8)$ \\
\hline
\end{tabular}

Scores are given in percentage. The number of considered 20-min epochs is denoted by $n$.

versus MABP, were statistically different ( $p \leq 0.01)$, with differences 9.3 and $5.8 \%$, respectively. Bland-Altman plots of the $\mathrm{COH}$ scores for the Leuven, propofol, and Utrecht datasets are shown in Figs. 2, 3, and 4, respectively.

Analysis including all the scores over all epochs. Table 3 shows the $p$-values pointing out the statistical significance of the differences in scores computed from $\mathrm{dHbD}$ (respectively, $\mathrm{dHbT}$ ) versus MABP (method 1) compared with cTOI (respectively, $\mathrm{rScO}_{2}$ ) versus MABP (method 2). Here, the scores are compared for each 20-min epoch. For each baby, multiple measurements are available. Generalized linear mixed models were used to assess the differences between both methods.
In the propofol dataset, the differences in $\mathrm{COR}$ and $\mathrm{COH}$ scores, computed from $\mathrm{dHbD}$ versus MABP compared with cTOI versus MABP, were not statistically significant $(p=$ 0.21 and $p=0.38$ ). Similar insignificant differences hold for the Leuven dataset ( $p=0.95$ and $p=0.08$ ).

For the Utrecht dataset, the differences in $\mathrm{COR}$ and $\mathrm{COH}$ scores, computed from dHbT versus MABP compared with $\mathrm{rScO}_{2}$ versus MABP, were statistically significant $(p<0.01$ in both cases), with differences 9.7 and $7.5 \%$, respectively.

Analysis including the scores over 20-min epochs with high variations in MABP. Table 4 shows a similar analysis as in Table 3, except that only 20-min epochs with a high 
variation in MABP $(>10 \mathrm{~mm} \mathrm{Hg})$ were retained for the calculations.

In the propofol dataset, the $\mathrm{COR}$ and $\mathrm{COH}$ scores, computed from $\mathrm{dHbD}$ versus MABP compared with cTOI versus MABP, were not statistically different $(p=0.96$ and $p=0.76$, respectively). Similar results hold for the Leuven dataset (with $p=0.57$ and $p=0.68$, respectively). Moreover, for the Utrecht dataset, the differences in $\mathrm{COR}$ and $\mathrm{COH}$ scores, computed from dHbT versus MABP compared with $\mathrm{rScO}_{2}$ versus $\mathrm{MABP}$, were no longer statistically significant (with $p=0.09$ and $p=0.21$, respectively).

For completeness of the study, we also used dHbT instead of $\mathrm{dHbD}$ in the whole analysis and comparison of both methods using the two cohorts of Leuven. No significant differences for the propofol dataset between $\mathrm{dHbD/MABP}$ and $\mathrm{dHbT} / \mathrm{MABP}$ were found. For the Leuven dataset, differences in mean $\mathrm{COH}$ score are significant $(p<0.008)$ and below $5 \%$, whereas differences in mean COR scores were larger (up to $17 \%$ ). These results are not included for conciseness of the article.

Analysis including mean scores per patient and all epochs per patient using data sampled at $3 \mathrm{~s}$. To study the influence of the sampling frequency on the scores, the differences in mean scores (COR and $\mathrm{COH}$ ), computed from $\mathrm{dHbD}$ versus MABP (method 1) compared with cTOI versus MABP (method 2), in the propofol and the Leuven datasets were analyzed on a patient level, and on epoch level. Although $p$-values were lower, similar conclusions hold as for the 60 -s data. Wherever differences were statistically significant, they always remained lower than $5.6 \%$.

\section{DISCUSSION}

Impaired cerebral autoregulation is considered a risk factor for brain injury in the sick premature infant $(2,10,12,23)$. However, previous studies using intermittent static measurements (5) showed that CBF is independent from MABP over a wide pressure range in premature babies. When there is a lack of autoregulation, oxygen delivery is a function of CBF and cerebral arterial oxygen content. Hence, it would be of clinical interest to have a continuous measure of autoregulation. Therefore, NIRS was used by Tsuji et al. (10). Tsuji et al. (11) and Soul et al. (30) validated $\mathrm{HbD}$ as a good measure of $\mathrm{CBF}$. Moreover, as total $\mathrm{Hb}(\mathrm{HbT})$ reflects changes in cerebral blood volume (CBV) (31) in patients with lack of autoregulative properties, as the ones included in this study, it is possible that $\mathrm{HbT}$ reflects $\mathrm{CBF}$. The main problem of these measurements is that they are very sensitive to movements and thus only applicable in research settings. More recently, spatially resolved spectroscopy introduced new parameters like cTOI and $\mathrm{rCCO}_{2}$, reflecting $\mathrm{Hb}$ oxygen saturation predominantly of the venous compartment (32). These parameters are less sensitive to movements and provide absolute values.

As shown in Tables 2-4, no important differences exist in using cTOI instead of $\mathrm{dHbD}$ and using $\mathrm{rScO}_{2}$ instead of $\mathrm{dHbT}$ for the measurement of autoregulation. The NIRS-derived parameter $\mathrm{dHbT}$ has been validated as a measure of blood volume, so it does not represent changes in blood flow.
Because data were obtained in stable infants, changes in $\mathrm{CBF}$ and CBV could be expected to be equivalent, as confirmed in our study. Significant differences, if any, in mean COR and mean $\mathrm{COH}$ scores between both methods are less than $7 \%$ when sampling the data at $60 \mathrm{~s}$, which is still within the normal variation induced for the NIRS parameters (13). Using $\mathrm{rScO}_{2}$ instead of $\mathrm{dHbT}$ as measure for autoregulation, larger differences (up to 9.7\%) were noticed. This can be produced for the difficulties in measuring $\mathrm{dHbT}$ using the INVOS4100 (this value is not user-accessible and was highly sensitive to artifacts) or because dHbT less reflects CBF compared with $\mathrm{dHbD}$ (11); however, there is no evidence to prove these assumptions. These differences can still be considered as normal, therefore, clinically unimportant when used, e.g. for detection of impaired autoregulation. However, all these differences become insignificant when only the larger variations in $\operatorname{MABP}(>10 \mathrm{~mm} \mathrm{Hg})$ are taken into account for the calculation of the $\mathrm{COH}$ and COR scores, as presented in Table 4. Small changes in MABP yield low values in their power spectral density. Because our $\mathrm{COH} / \mathrm{COR}$ score calculations are using spontaneous MABP changes, a lot of which are small, these might affect their reliability as confirmed by Hahn et al. (33). Therefore, these epochs of higher MABP variations enable a more reliable detection of (im)paired autoregulation. They better assess the autoregulative properties of the brain and are clinically more important.

Even though there are no important statistical differences between the methods, large discrepancies in the COR and $\mathrm{COH}$ values on an infant-to-infant basis exist, as depicted in the Bland-Altman plots (Figs. 2-4). Hence, the agreement is not very high either. These discrepancies, as mentioned before, can be attributed to the normal variations of the NIRS parameters and to the fact that model assumptions are only approximately valid in practice. The NIRS parameters only indirectly measure $\mathrm{CBF}$ and their coupling with MABP approximately reflects autoregulation assuming $\mathrm{SaO}_{2}$ constant, which is only approximately valid. More importantly, what really matters here is the lower sensitivity of cTOI and $\mathrm{rScO}_{2}$ to movement artifacts compared with $\mathrm{dHbD}$ and $\mathrm{HbT}$. Therefore, they represent a more robust variable to assess the autoregulation status of the infants.

Hence, this enables the direct clinical use of signal processing methods for the automated and continuous calculation of the coupling between $\mathrm{dHbD} / \mathrm{cTOI}$ and $\mathrm{dHbT} / \mathrm{rScO}_{2}$ versus MABP, using NIRS, mostly by means of $\mathrm{COR}$ and $\mathrm{COH}$. However, these scores are not a precise measurement of autoregulation but rather a reflection of the autoregulation status of the baby.

It is important to recognize here the potential limitations of the use of $\mathrm{COR}$ and the $\mathrm{COH}$ analysis to investigate momentto-moment autoregulation mechanisms, because these approaches assume a linear and stationary relationship between the measured NIRS signals and MABP, which can produce misleading results in a system with nonlinear and nonstationary properties. Future research should be oriented to evaluate these effects.

Finally, the scores should be related to clinical outcome of the infants. The scores of our propofol dataset are shown to be 
related to clinical intervention because they change concordant to the administration of propofol (18). No other conclusions can be drawn because the populations used here are too small. However, this is part of ongoing research, and the results will be published elsewhere.

\section{CONCLUSIONS}

Little or no statistical differences are found between mean $\mathrm{COR}$ and mean $\mathrm{COH}$ scores computed from $\mathrm{dHbD}$ versus MABP compared with cTOI versus MABP using NIRO300 recordings from Leuven and between scores computed from $\mathrm{dHbT}$ versus MABP compared with $\mathrm{rScO}_{2}$ versus MABP using INVOS4100 recordings from Utrecht, although large discrepancies exist on an infant-to-infant basis. Using three different datasets, recorded in two different centers Leuven and Utrecht with two different devices and sampled at two different rates 3 and $60 \mathrm{~s}$, we showed a similarity in scores by replacing the measured NIRS signal $\mathrm{dHbD}$ by cTOI when using a NIRO300 instrument and by replacing $\mathrm{dHbT}$ by $\mathrm{rScO}_{2}$ when using an INVOS4100 instrument. Hence, cTOI and $\mathrm{rScO}_{2}$ can be used for the calculation of cerebral autoregulation in neonates. Moreover, as the frequency range is restricted to lower frequencies (smaller than $0.008 \mathrm{~Hz}$ ), we showed that the correspondences between the scores derived from $\mathrm{dHbD}$ and cTOI or $\mathrm{dHbT}$ and $\mathrm{rScO}_{2}$, increase. These correspondences further increase when restricting the $\mathrm{COH} /$ COR score calculations to those epochs with large enough variations in MABP $(>10 \mathrm{~mm} \mathrm{Hg})$.

The next step will be to generate software to use these parameters online in patients and to study the autoregulation in different situations in clinical practice. The importance of these findings is that we now have a reliable monitor to measure cerebral autoregulation noninvasively and continuously in preterm infants. Whether this parameter can help us in treating patients and prevent cerebral complications will have to be studied in the future.

\section{REFERENCES}

1. Lassen NA 1959 Cerebral blood flow and oxygen consumption in man. Physiol Rev 39:183-238

2. Greisen G 2005 Autoregulation of cerebral blood flow in newborn babies. Early Hum Dev 81:423-428

3. Pryds O 1991 Control of cerebral circulation in the high-risk neonate. Ann Neurol 30:321-329

4. Dempsey EM, Barrington KJ 2007 Treating hypotension in the preterm infant: when and with what: a critical and systematic review. J Perinatol 27:469-478

5. Tyszczuk L, Meek J, Elwell C, Wyatt JS 1998 Cerebral blood flow is independent of mean arterial blood pressure in preterm infants undergoing intensive care Pediatrics 102:337-341

6. Panerai RB, Kelsall AW, Rennie JM, Evans DH 1995 Cerebral autoregulation dynamics in premature newborns. Stroke 26:74-80

7. Panerai RB, Kelsall AW, Rennie JM, Evans DH 1996 Analysis of cerebral blood flow autoregulation in neonates. IEEE Trans Biomed Eng 43:779-788

8. Rennie JM 1998 Autoregulation of cerebral blood flow. Lancet 352:2023
9. Steiner LA, Pfister D, Strebel SP, Radolvich D, Smielewski P, Czosnyka M 2009 Near-infrared spectroscopy can monitor dynamic cerebral autoregulation in adults. Neurocrit Care 10:122-128

10. Tsuji M, Saul JP, Duplessis A, Eichenwald E, Sobh J, Crocker R, Volpe JJ 2000 Cerebral intravascular oxygenation correlates with mean arterial pressure in critically ill premature infants. Pediatrics 106:625-632

11. Tsuji M, Duplessis A, Taylor G, Crocker R, Volpe JJ 1998 Near infrared spectroscopy detects cerebral ischemia during hypotension in piglets. Pediatr Res 44:591595

12. Wong FY, Leung TS, Austin T, Wilkinson M, Meek JH, Wyatt JS, Walker AM 2008 Impaired autoregulation in preterm infants identified by using spatially resolved spectroscopy. Pediatrics 121:e604-e611

13. van Bel F, Lemmers P, Naulaers G 2008 Monitoring neonatal regional cerebral oxygen saturation in clinical practice: value and pitfalls. Neonatology 94:237-244

14. Suzuki S, Takasaki S, Ozaki T, Kobayashi Y 1999 A tissue oxygenation monitor using NIR spatially resolved spectroscopy. Proc Soc Photo Opt Instrum Eng 3597:582-592

15. Kim MB, Ward DS, Cartwright CR, Kolano J, Chlebowski S, Henson LC 2000 Estimation of jugular venous $\mathrm{O} 2$ saturation from cerebral oximetry or arterial $\mathrm{O} 2$ saturation during isocapnic hypoxia. J Clin Monit Comput 16:191-199

16. Nagdyman N, Fleck T, Schubert S, Ewert P, Lange PE, Abdul-Khaliq H 2005 Comparison between cerebral tissue oxygenation index measured by near-infrared spectroscopy and venous jugular bulb saturation in children. Intensive Care Med 31:846-850

17. Daubeney PE, Pilkington SN, Janke E, Charlton GA, Smith DC, Webber SA 1996 Cerebral oxygenation measured by near-infrared spectroscopy: comparison with jugular bulb oximetry. Ann Thorac Surg 61:930-934

18. Vanderhaegen J, Naulaers G, Van Huffel S, Vanhole C, Allegaert K 2010 Cerebral and systemic hemodynamic effects of intravenous bolus administration of propofol in neonates. Neonatology 98:57-63

19. Benaron DA, Kurth CD, Steven JM, Delivoria-Papadopoulos M, Chance B 1995 Transcranial optical path length in infants by near-infrared phase-shift spectroscopy. J Clin Monit 11:109-117

20. Wyatt JS, Cope M, Delpy DT, Van der Zee P, Arridge S, Edwards AD, Reynolds EO 1990 Measurement of optical path length for cerebral near-infrared spectroscopy in newborn infants. Dev Neurosci 12:140-144

21. de Waal EE, De Vries JW, Kruitwagen CL, Kalkman CJ 2002 The effects of low-pressure carbon dioxide pneumoperitoneum on cerebral oxygenation and cerebral blood volume in children. Anesth Analg 94:500-505

22. von Siebenthal K, Beran J, Wolf M, Keel M, Dietz V, Kundu S, Bucher HU 1999 Cyclical fluctuations in blood pressure, heart rate and cerebral blood volume in preterm infants. Brain Dev 21:529-534

23. Soul JS, Hammer PE, Tsuji M, Saul JP, Bassan H, Limperopoulos C, Disalvo DN, Moore M, Akins P, Ringer S, Volpe JJ, Trachtenberg F, Duplessis AJ 2007 Fluctuating pressure-passivity is common in the cerebral circulation of sick premature infants. Pediatr Res 61:467-473

24. Caicedo A, Van Huffel S 2010 Weighted LS-SVM for function estimation applied to artifact removal in biosignal processing. Presented at: Engineering in Medicine and Biology Society (EMBC), 2010 Annual International Conference of the IEEE, IEEE, Piscataway, New Jersey, pp 988-991

25. Suykens JA, Van Gestel T, De Brabanter J, De Moor B, Vandewalle J 2005 Least Squares Support Vector Machines. World Scientific, Singapore, pp 98-100

26. Proakis JG, Manolakis DG 1996 Digital Signal Processing. Prentice-Hall, Upper Saddle River, pp 910-913

27. Brun NC, Moen A, Borch K, Saugstad OD, Greisen G 1997 Near-infrared monitoring of cerebral tissue oxygen saturation and blood volume in newborn piglets. Am J Physiol 273:H682-H686

28. Leuridan J, Rost B 1985 Multiple Input Estimation of Frequency Response Functions: Diagnostic Techniques for the Excitation. American Society of Mechanical Engineers 85-DET-107. American Society of Mechanical Engineers, New York, NY

29. Morren G, Naulaers G, Lemmerling P, Van Huffel S, Casaer P, Devlieger H 2003 Quantitation of the concordance between cerebral intravascular oxygenation and mean arterial blood pressure for the detection of impaired autoregulation. Adv Exp Med Biol 510:403-408

30. Soul JS, Taylor GA, Wypij D, Duplessis AJ, Volpe JJ 2000 Noninvasive detection of changes in cerebral blood flow by near-infrared spectroscopy in a piglet model of hydrocephalus. Pediatr Res 48:445-449

31. Wyatt JS, Cope M, Delphy DT, Richardson CE, Edwards AD, Wray S, Reynolds EO 1990 Quantitation of cerebral blood flow volume in human infants by near-infrared spectroscopy. J Appl Physiol 68:1086-1091

32. Kurth CD, Uher B 1997 Cerebral hemoglobin and optical pathlength influence near-infrared spectroscopy measurement of cerebral oxygen saturation. Anesth Analg 84:1297-1305

33. Hahn GH, Christensen KB, Leung TS, Greisen G 2010 Precision of coherence analysis to detect cerebral autoregulation by near-infrared spectroscopy in preterm infants. J Biomed Opt 15:037002 